\title{
Technology transfer a global perspective: Crux of the matter
}

\author{
Ramesh S. Yamgar ${ }^{1}$, Sudhir S. Sawant ${ }^{2, *}$ \\ ${ }^{1}$ ICLES's, Motilal Zhunzhunwala College of Arts, Commerce and Science, Vashi, Navi Mumbai 400 703, INDIA \\ ${ }^{2}$ P. G. Dept. of Chemistry, Govt. of Maharashtra, Ismail Yusuf Arts, Science and Commerce College, Jogeshwari, Mumbai 400060 INDIA
}

Email address:

sawantsudhir@hotmail.com (S. S. Sawant)

To cite this article:

Ramesh S. Yamgar, Sudhir S. Sawant. Technology Transfer a Global Perspective: Crux of the Matter. Science Research. Vol. 2, No. 3, 2014, pp. 27-37. doi: 10.11648/j.sr.20140203.11

\begin{abstract}
Technology transfer has become a key activity in the pharmaceutical manufacturing domain. The processes are developed in an R\&D set up for the API or finished dosage forms and are transferred to another site in the same country or different geographic location. Due to changing business needs, technology transfer has become a global activity and opens up various opportunities for the professionals in pharmaceutical domain. The coordination of various linked activities like procurement, quality, production, health \& safety of the personnel involved in manufacturing and export have become more vital. This article discuss about various aspects of technology transfer like outsourcing key raw materials, information security to protect the intellectual property, various development strategies for API development and most significantly the practical difficulties encountered during API technology transfer. It is also describing certain trouble shooting issues, which were resolved with the help of reaction mechanism \& chemistry involved in processes and investigations made to arrive at appropriate CAPA. This article is also indicating simple laboratory techniques/ instruments, which could be adopted to support PAT initiatives. It is also describing examples of how certain process improvements were achieved due to trouble shooting in scale up trial batches.
\end{abstract}

Keywords: API Technology Transfer, Information Security, Process Trouble Shooting, Process Validation \& DMF Filing, PAT, CAPA

\section{Introduction}

In view of the ever increasing overhead costs in the Europe due to various economic reasons and less availability of trained manpower, many European organizations are looking at India as next destination for their organic growth. Indian government policies to encourage foreign direct investment in India and creation of SEZ for industrial growth in not so developed areas of Indian continent, makes European companies to look at India as an opportunity towards establishing manufacturing sites in India. India also has other subtle advantages compared with other Asian countries with respect to availability of well educated and trained man power. With the number of USFDA approved manufacturing sites all over India, the man power is well acquainted with regulatory requirements of manufacturing pharmaceutical products. Also increasing awareness about health, safety and environment protection practices among Indian pharmaceutical organizations make India a more attractive business partner for European organisations. India's economic policies and availability of huge talent pool for sustaining and growing operations are making India an attractive choice for global pharma companies for investment, tie-ups, mergers and acquisitions [1]. India has proven international quality standard capabilities as measured by ever increasing number of ANDA approvals, DMF filings, US FDA/ UK MHRA approved manufacturing facilities/ bio equivalence centers, which are considered as key indicators for assessing the capabilities of any national pharma sector.

It is necessary to understand the overall global trend that is being observed over last couple of years. It is now clear that the pipe line for new chemical entities by innovator pharma companies is getting dried up year after year. The success rate of high throughput screening and parallel synthesis and biotechnology related breakthroughs still remains a dream for major innovator organizations. The ever rising costs of discovering a new medicine and failures 
of new drug candidates at advanced clinical trials make entire process very much uncertain and cost intensive.

India being a major generic player since 1980's, has mastered certain technologies over other asian competitors like China. Increasing population of English speaking people and with the advent of new information technologies like internet, entire world is coming closure from the communication perspective. Use of video conferencing and other telecommunication channels over the internet also adds to these distances being shortened. There exists no much need to go personally overseas just for attending a simple meeting. Most the discussions can be now completed using web meetings, which not only saves money, but time and efforts of unnecessary and too much tiring international travel. A group of people across the various sites now can sit comfortably in their respective offices, get connected through web meetings and can have a detailed discussion regarding any specific matter. This is improving the team building and team spirit towards achieving a common objective. Such meetings also enhance the confidence of the cross functional team members towards achieving a set target.

Although numerous books and hundreds of articles have been written on technology transfer practices, hardly any paper deals with the real issues faced during technology transfer. Objectives and requirements of technology transfer process are normally found to be varying from stage to stage, which altogether makes the technology transfer process more difficult and complex to get materialized [2].

\section{Outsourcing Activities}

With increasing investment in India to set up state of the art manufacturing units, India would become a global outsourcing and manufacturing hub for major pharmaceutical companies. The number of manufacturing units will become more compared to basic research and development units. Revenues will be generated and much of foreign exchange would be earned for the India through export of pharmaceutical products. To support such manufacturing processes, technology transfer from global research and development units to Indian manufacturing units would become inevitable. Technology transfer teams would play an important role in translating small scale R\&D process knowledge into large scale manufacturing activities. Process development and technology transfer would become the most important bridge between global research units to receiving sites for manufacturing on bulk scale. Hence conscious efforts towards building such cross functional team is an important area of human resource development. People need to be trained towards multidisciplinary and multitasking objectives. Here the "first time right" approach carries a lot of significance. If a certain activity is completed right first time, it saves a lot of time, money and efforts at the end. Such initiatives need encouragement, support and facilitation from the senior management.

Technology transfer is a misnomer in pharmaceutical field. Rather than visualizing technology transfer in discrete stages, it should be considered with a holistic view [3]. Technology is often related to very large scale manufacturing, for examples, production of petroleum products from crude oil. In this case distillation technology is used to manufacture purer liquid products like Toluene. Other example can be production of methanesulphonic acid using very special technology by BASF. Yet other examples of technology products in manufacturing on large scale products like methylamine, dimethylamine, trimethylamine using basic raw materials like methnol solvent, ammonia gas and solid phase catalyst under very high temperature and pressure. Most simplest example, can be manufacturing of urea by Haber process [4] from carbon dioxide and ammonia with a distinct cost advantage. These are often continuous fed processes and not batch processes.

\section{Information Security}

Information security related issues are among the most challenging tasks during transfer of knowledge from a research and development unit to a manufacturing facility. India being a member to WIPO and respects intellectual property or patents rights, concerns about maintaining secrecy of certain processes are often raised. However, such issues may be dealt with by adopting simple and cost effective information security policies.

Information or knowledge available in public domain cannot be patented. However novel processes, new knowledge, new ways of working and new ideas can be protected by patents. Circulation or sharing of such knowledge to limited number of key people could be the first step towards practicing such sensitive processes. Such information may not be shared by email, if shared, such emails cannot be forwarded or have an expiry date after which such emails get deleted automatically. Files containing such knowledge may not be saved or printed or attached to other emails and have specific administrative rights to sender/originator. Sensitive data could be stored in a common drive on network server and could be assessed as and when required by specific group of people, such access can be limited by providing access login ids and passwords. User can only read the knowledge, may not be able to print or download such files from common server. Such downloading may be allowed in certain cases depending on priority and need. There can be coding of important catalysts for novel process right from warehouse stage. In short by adopting simple strategies, information or knowledge of an organization can be protected. However while adopting such policies, care should be taken to also find out practical ways of working in manufacturing shop floor. Everything cannot be coded, as it does not give confidence to shop floor person in the beginning and to an auditor at the end. 


\section{Strategies}

Among various business strategies adopted by pharmaceutical organizations, following development models could be pursued, but not limited to only these. There could be combinations of these and many more like these may exists. We are just attempting to give an overview of what we encountered so far in our professional experience.

$>$ Development Model 1

This is model of development is the most systematic activity through research development laboratories. Typically, it starts with a project being shortlisted by project port folio management committee. This decision is mainly based on the forecast of the demand and supply requirements of an API or pharmaceutical product. Litrature survey is thoroughly done using advanced literature search tools like Scifinder for patents and publication regarding processes and to mainly understand current patent situation. Based on the patent summary report, new non-infringing routes are thought and more than one synthesis routes are tried out on a small scale in synthesis laboratories. Depending on the success of such synthesis procedures, availability of key starting materials, reagents, these routes are further scaled up in kilo labs to get more confidence on the process, safety and quality aspects. Stability studies, holding time studies, forced stability studies are done at this stage on APIs generated at kilo scale. After the kilo lab campaign, pilot plant scale batches are planned and further confidence is gained on the process. Any scale up related issues is addressed properly without any ambiguity and the process is ready for real transfer to manufacturing scale. All the data from the lab scale, kilo scale and pilot scale is gathered in the form of a development report, scale up report and shared with cross functional team during tech transfer meeting/s and with manufacturing plant.

QbD: Quality by Design process. Process is designed not to fail in either in quality or cost. Good balance is required between two important factors for commercial success of an API project.

$>$ Development Model 2

This model is adopted by organizations which are having late entries in generic market and want to have quick success with respect to return on capital investment. Often R\&D scientists are given less than three months time to about six month time depending on the number of steps in synthesis to develop a process. The strategy is simple to copy certain base patents or other patents and have a working process for scale up in manufacturing plant. More inclination is towards copying the patent typical examples as it is without knowing that these are just examples for applying for patents and not real processes for manufacturing purpose. It is said that "patents are not technology transfer documents", this emplies above fact that real processes for manufacturing are developed at later stage, which probably are not disclosed in full extent in public domain. Another strategy is to face trouble shooting during scale up campaigns and make working procedures to solve the issues faced and go ahead with such half cooked processes for validation campaigns. Often validation campaigns are managed by research and development department in this fashion and DMFs are filed on false sense of urgency. Such DMFs also get approved by regulatory agencies subsequently and now these processes are ready for commercialization. Here the real trouble starts, as it is now the complete responsibility of production department to produce the required commercial quantities. The first post validation batch attempt is a failure with respect to quality or cost. Quality deviations need thorough investigations and CAPAs are proposed to address quality issues. Such CAPAs are inadequate and often not implementable on routine basis in production. In second attempt, again quality issues are faced and it is not possible to produce and dispatch required API quantities to either internal customer like formulation department or an external customer. These overall results in losses with respect to time, money and efforts of everyone involved and project being not realized. The approximate cost of filing one DMF by an Indian industry is in tune of ten million rupees (i.e. one crore rupees). Such an investment is not able to fetch returns on capital invested. Frequent quality issues, ultimately results in project being dropped out or closed. It may be sent back to research and development for redeveloping new process or new route depending upon the commercial viability of generic molecule.

\section{$>$ Development Model 3}

This model is also adopted by certain organizations, in which, not much time is spent in research and development efforts and half cooked processes or processes copied from other manufacturers/ patents are directly attempted on pilot or manufacturing scale. This strategy is to save time and invest money in trial and error efforts, face troubleshooting on big scale, make workable processes to manufacture and supply APIs to non-regulated or domestic markets through the processes developed in this not so scientific manner. In this model it is a costly affair, but time saving and can deliver faster to market demands and customer requirements. This can bring quick wins in generic market. These organizations do not file DMFs, as their business plans are not for regulated markets, but for under developed countries and domestic or semi-regulated markets. An estimate indicates that non-regulated market is much bigger in size and volume than the regulated markets of US, Europe and Japan taken together.

\section{Practical Difficulties during Transfer of Technology}

In this article, we would like to highlight the major issues faced by pharmaceutical organizations, their probable route causes and possible solutions to overcome 
these issues partially and not completely. Issues can be reduced but cannot be completely eliminated. Chemistry is a well understood and predictable science. Without completely understanding the chemistry involved, it is unreasonable to expect that chemistry will deliver desired results in a commercial domain. Here benchmarking i.e copying the best practices of other industries, setting of new mile stones and paradigm shift i.e. "complete shift in thinking process" is necessary. Traditional thinking blended with modern thinking approach is necessary for converting many uncertainties into realities.

In pharmaceutical technology transfer and manufacturing, these are batch processes and not continuous processes. Often they are small in input and output volumes, as requirements are not large and limited to certain part of year on need basis. Continuous production is not required to be done, only campaign basis production is necessary.

In this context, technology transfer is knowledge transfer from research and development know how at small scale in laboratory, thinking how to scale up these experiments, what imagination required, what difficulties may be encountered during such exercise and "how to do 'right first time' approach". This is related to following soft activities often hidden, but absolutely essential for a successful and complete technology transfer.

1. Process chemistry knowledge transfer : critical process parameters, critical quality attributes, process flow, equipment train for scale up, in process controls, formation of impurities, depletion/ purge of impurities, reworking and reprocessing procedures, holding points and holding time studies, stability of wet cakes of intermediates, heat stability of intermediates and APIs during drying, hygroscopicity of the intermediates and APIs, light sensitivity of reaction mixture, intermediate or API.

2. Analytical knowledge transfer: analytical development in research lab to quality control lab, set procedures, no scope to change any parameter once transferred to receiving site, SOPs, audits etc. Methods of analysis, impurity standards, reference and working standards, raw material specifications, setting of "in process" analysis specification limits, intermediate analysis specifications, final API analysis specifications, justification of specifications, stability testing, stress stability data, shelf life of intermediate and API.

3. Commercial knowledge at research and development: transfer to manufacturing scale, raw material availability, lead times for delivery at site, purity issues, tight specifications, uncertainty to get such specialty chemicals, costs, other factors like transportation, overseas manufacturing sites of certain critical raw materials, storage conditions. Warehouse facilities to store at low temperatures. Solvent storage and dispensing, losses of solvents due to higher temperatures in summer.

4. Solid state analytical knowledge /physical chemistry knowledge transfer: heating rates, cooling rates, surface areas, mass flows, vacuum requirements, drying time etc. Reaction exothermicity, heat of reaction, adiabatic temperature rise, runaway situations etc.

5. Equipments in manufacturing plant:

i. Equipment train: It is absolutely essential to understand what type of production equipments are required to carry out certain process in manufacturing plant. One has to understand the MOC i.e. material of construction, minimum and maximum stirrable volume of a reactor, minimum and maximum sensing volume of reactor, what type of utilities are necessary during batch operations like cooling, heating, distillation etc. One has to also decide on the process flow and how many equipments are required for a validation campaign and accordingly $\mathrm{BMR}$ is written. How many lots may be required to make to centrifuge the wet cake of material, how one can ensure uniform washings to all lots, how to unload wet cake and charge in drying equipment. How to unload dried material from vacuum oven, RCVD or ANFD etc. in a safe way without too much of exposure to shop floor personnel.

ii. Cleaning validation: Cleaning of process equipments is also an essential part of validation campaign. It is necessary to identify good organic solvents during development stage to clean equipments in manufacturing plant. It is possible sometimes, to clean using only water, due to good solubility of intermediates or APIs in water. Often a combination of water and organic solvent like methanol is considered. Detergents are also used for cleaning purpose. Equipments like CIP are used to save on quantity of solvents used for cleaning. Suitable analytical methods are developed to verify cleaning results and ensure documentary evidence that cleaning was effective and possibility of carry over or contamination to other product was eliminated. In samples of cleaning, usually API concentration of $10 \mathrm{ppm}$ or below is expected for cleaning validation analysis. However, developing analytical methods which can detect such low concentrations is a challenge. High potent APIs also need to be thoroughly cleaned and cleaning validation should be completed successfully. In these cases, analytical methods must be able to detect in low ppm or even at ppb concentrations. MACO calculations are done to understand maximum allowable carry over concentration of APIs [5].

6. Reaction and Process safety knowledge: It is necessary to understand the nature of reaction by reaction calorimetry. In case of an exothermic reaction, how much is adiabatic temperature rise? It helps to understand the risky operations and to decide on 
control mechanism. Additional safety studies like mixing experiments, compatibility testing, powder handling studies like falling hammer test, autoignition temperature or dust explosion experiments would give more information with respect to process safety. Exothermic reactions often can be controlled through slow addition of reagents, or diluting reagent with suitable solvent and also incorporating other engineering controls like low temperatures in reactor jackets etc. It is necessary to generate safety data of potential hazardous reactions including reaction class $1,2,3$, Occupational exposure band(OEB)[6], LD $_{50}$ values of high potent APIs, containment systems for toxic chemicals, intermediate or APIs and safe disposal of toxic waste/ mother liquors.

7. Environmental impact: Chemistry is no more a dirty domain. Professionally it can be made into a clean and sustainable domain. Those who cannot maintain clean chemistry are not true chemists and they are unprofessional in chemistry domain. It is obligatory to control gaseous emissions or reduce their direct release into atmosphere. By adopting green chemistry approach, many hazardous operations involved in scaling up processes can be mitigated. Let us do not talk big things, instead act small things, as an ounce of practice is better than a tons of theory. Solvent recovery and reuse, regeneration of catalyst, recycling mother liquors are some of the areas for improvements and reduction of environmental pollution. Adopting alternative reagents having less environmental impact and reducing effluent load are the keys to which follow green chemistry initiatives.

8. Commercial aspects: Cost reduction may not be goal of technology transfer in initial phases, however developing low cost processes or alternative technologies may come under purview of technology transfer. Raw material cost reduction has no much scope as the prices of chemicals are more or less same throughout the world. Hence focusing on isolation of second crops from mother liquors, solvent recovery and reuse, catalyst recycling and reuse, judicious use of utilities and project planning and execution would be some of the important commercial aspects during technology transfer.

9. Planning and coordination across CFT members: The first coordination begins with procurement team to get chemicals timely on site. Some of the chemicals may have to be imported and may have long lead times to arrive by sea route. Common understanding of the process requirements with respect to warehouse for storage of chemicals, transportation of finished products, coordination with QC for analytical method transfers, procurement of HPLC columns, equipments required for analysis, raw material analysis and release of intermediates for further manufacturing steps, coordination with quality assurance for documents, statutory requirements and validation master plan, coordination with production to understand equipments, accessories, utility requirements, safety awareness and training of shop floor personnel, coordination with EHS for hazardous operations, mitigation of risks and preparedness for emergency situations like fire, injuries etc. coordination with liaison department for timely receipt of manufacturing licenses from government authorities, regulatory agencies etc.

10. Project management: It is not the strongest who survives, but the most adoptable to change, most lean among the others, is the one who is going to survive for longer in any business scenario. An efficient project manager involves everyone responsible for the decisions and has a participative style of working. Project manager maintains a high level of professional integrity and transparency across all CFT members. He can use modern tools like MS Office Project software to map entire gamut of operations and activities for a successful technology transfer. In project management concept, technology transfer could be one of the mile stones of entire project. Other mile stones like launching the product in specific market could be the biggest task to accomplish.

11. Attrition rate: employees leaving organizations quite early in life cycle of product, the expertise developed inhouse being eroded and not replaced immediately with new set of skilled manpower. Often gaps are created as long as process understanding and process controls with respect to quality and safety are concerned. Hence often post-validation campaigns face a lot of hiccups, failures and this may result in major deviations with respect to quality and cost. Certain project may not be viable due to increased cost of production. Hence retention of employees must be an important agenda of human resource department of any organization.

12. Packaging and transporting: Often APIs need to be transported at low temperatures. This could be a challenge at temperate zones like Asia, Africa \& Middle east. Innovative packaging techniques needs to be developed, which can ensure low temperature transport and also monitor temperatures using sensor and data loggers[7].

\section{Difficulties Faced during Transfer of Processes to Manufacturing Plant}

1. Availability of raw materials with tight specifications of purity, assay or water content limits. Delivery times, transport conditions, storage and chemical stability issues: Solvents like ethanol, methanol and DMF tend to absorb moisture and it is not practically possible to have very less moisture content i.e. less than $0.1 \%$ (1000ppm). So very tight specification of water content, less than $1000 \mathrm{ppm}$ in such solvent is very 
difficult to get on commercial large scale. Sometimes, chemistry demands very very low moisture content levels which cannot be met with commercial supplies. In such cases, solvents having about $0.2 \%$ to $0.5 \%$ moisture content should be treated "in house" with molecular sieves prior to use. Molecular sieves powder could be added, stirred and solvent filtered or molecular sieves could be packed into an SS column and solvent is circulated using a pump through this column till the moisture content upto certain low ppm level ( typically upto 100 to $300 \mathrm{ppm}$ ) is achieved. Karl Fischer Coulometer should be used to detect such low moisture content levels in ppm levels.

2. Raw material analysis and release by QC. Troubles are often faced by quality control analyst during analysis of raw materials. These are due to incomplete understanding of the method of analysis and communication gap between Analytical research and development method transfer and receiving end in quality control. Here the quality assurance plays important role to minimize the gap between two sites and also technology transfer analytical chemist can bridge the gap between two sites. Technology transfer package should contain typical chromatograms and joint analysis exercise should be done using analytical R\&D scientist and QC chemist to develop confidence of analysts involved. This is also a part of QC personnel training and validation. Basically sample preparation skill and method knowledge transfer is required during such attempts.

3. Dispensing of critical raw materials, hazardous chemicals like pyridine borane, n-buLi, pyrophoric materials like dibal-H, weighing in warehouse. On line weighing load cells in manufacturing block necessary to view online rate of addition/ dosing of a liquid reagent. This is a good control on the process and ensures exact dispensing and amount being added to a reactor.

4. In process analysis, reproducibility issues, if does not comply, what next??? No clear instructions in BMR. For example, If $\mathrm{pH}$ goes beyond the specified limit, which reagent, and in what dilution should be used to adjust $\mathrm{pH}$ within specified range. Such instructions are expected from $R \& D$ scientist. In certain, "in process controls tests" like, $\mathrm{pH}$ adjustment, there are no clear instructions given in the process, if $\mathrm{pH}$ goes beyond specified limit. Hence, manufacturing chemist gets confused about what to do next? Which reagent should be used to adjust $\mathrm{pH}$, dilute hydrochloric acid, concentrated hydrochloric acid or acetic acid? If $\mathrm{pH}$ to be adjusted in alkaline range, which reagent, $10 \%$ sodium hydroxide solution or $1 \%$ sodium hydroxide solution or aqueous ammonia solution to be used ? What if, $\mathrm{pH}$ does not stay stable, how long one should wait to stabilize the $\mathrm{pH}$. In other troubles like, content of starting material by HPLC, does not show within specified limit and remains unreacted, what actions are required to be taken. These instructions should be mentioned in process and in BMR to take right decisions during progress of manufacturing batch. If clear information and instructions are missing, then batch may remain on hold until decision from $R \& D$, and it may deteriorate further with respect to quality and yield. If decisions are not taken immediately, entire batch may degrade ending up with no product at all and complete batch failure may occur. This results in deviation and further investigations and corrective and preventing actions. Hence, it is essential that R\&D has complete information of the process compiled in the form of a process development report and it is shared with manufacturing site for easy reference and for thorough understanding of the process parameters. Typical chromatograms of "in process HPLC tests", should be included in the report for comparison with QC chromatograms. The retention times and RRTs of impurities should be mentioned. Blank peaks arising due to mobile phase or gradient runs should be mentioned in the HPLC method of analysis.

5. Extended reaction times: In manufacturing scale, many times, reactions do not complete in time frame as per R\&D experimental data. Sometimes this could take longer than usual time for completion. In such extended time, what is impact on impurity generation, product getting degraded due to extended time etc., information comments/need to be included in process development report.

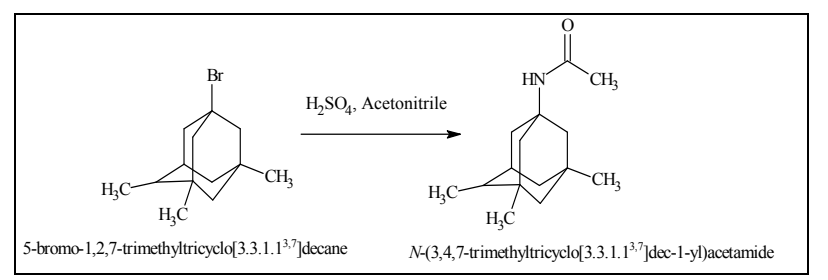

Figure 1. Conversion of bromo decane intermediate to acetamide intermediate.

In one of the modified Ritter reaction ( Figure 1), bromo decane intermediate was converted into acetamide intermediate using sulphuric acid as solvent and acetonitrile as reagent ( refer to mechanism of Ritter reaction). In typical R\&D experiment, bromo decane intermediate was monitored by GC for its conversion to acetamide intermediate. In process limit was not more than $10 \%$ after 18 hours of stirring at room temperature. In process data of R\&D experiments showed $5 \%$ to $8 \%$ unreacted bromodecane. In scale up trial batch, reaction was stirred in a glass line reactor for 18 hours as per $R \& D$ procedure, and in process test GC graph, showed no bromodecane peak, indicating complete conversion of starting material. However, the product peak height and size was very small. After workup the yield obtained was very less, nearly $20 \%$ compared to that of $80 \%$ for R\&D experiments. Intermediate analysis showed an unknown impurity of 
about $0.5 \%$, limit being not more than $0.20 \%$. This resulted in a deviation with respect to quality and yield. The cost also went up by four times and it was a matter of further investigation to find out suitable CAPA.

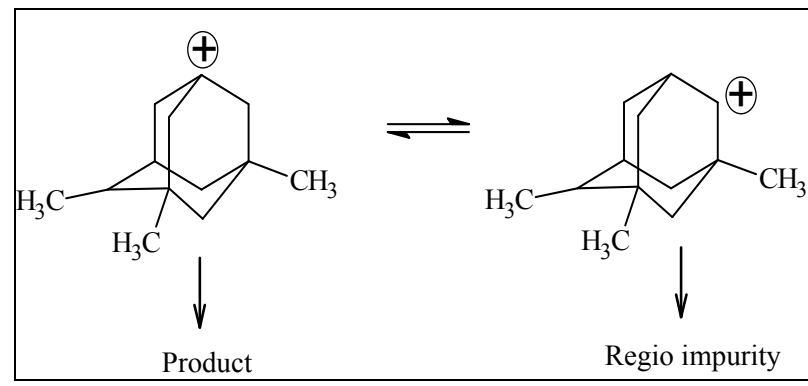

Figure 2. 1,2 shift of carbocation.

Initially, as part of investigation, no assignable cause was found at the manufacturing site and hence matter was referred to $R \& D$ for further investigation. In $R \& D$, impurity was isolated by recrystallisation technique. Proton NMR showed similar set of signals and same mass [M+1] peak. R\&D concluded that the impurity was a regio isomer due to isomerisation of carbocation [ Figure 2] formed after bromine atom departure. Cause of low yield, was assigned to decomposition of product due to extended stirring in highly acidic medium of sulphuric acid. Most of the molecular structure being aliphatic in nature, was getting decomposed and these degradation by- products were not observed in GC chromatogram. Later on, it was found that reaction completes in about 6-8 hours in glassline reactor. This was due to an efficient stirring of reaction mass by propeller type of stirrer blade. In R\&D, a simple Teflon blade was used, which did not mix reaction mass effectively, hence required longer time i.e. 18 hours for completion.

6. Sometimes, it is observed that data are not available with R\&D and in that case, small scale experiments can be performed to generate data and gain more insights into reaction progress, holding time data, quality aspects of the intermediate due to holding, wet cake stability data. "What if" type of experiments could be performed and data generated may be used to understand process as in-depth as possible.

7. Impurities formation: In an interesting investigation, about in process test deviation and later on intermediate quality deviation, by product formation was discovered. Reaction was formation of secondary amine intermediate as shown in Figure 3. It is an aldehyde condensation with benzyl amine to form an "in situ" imine and then hydrogenation of imine to secondary amine intermediate. As per R\&D, formation of imine was an instantaneous reaction and does not require longer digestion time.

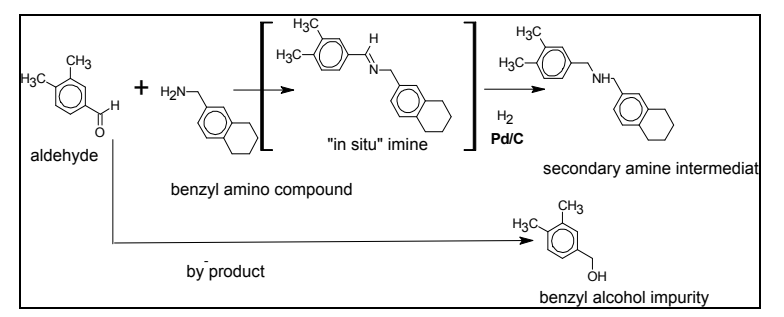

Figure 3. Generation of by product due to incomplete imine formation.

Scale up trial batch in pilot plant on a scale of $200 \mathrm{Kg}$ input of aldehyde was charged. After adding both reactants i.e. aldehyde and benzyl amine in a pressure reactor, $\mathrm{Pd} / \mathrm{C}$ catalyst was charged and hydrogenation was carried out. However, in process, HPLC graph, showed an extra peak due to some unknown impurity. Reaction was continued for additional 8 hours with intermittent check for progress of reaction by HPLC. However, extra peak did not deplete and it was an "in process test" deviation.

As part of investigation, the impurity was isolated by column chromatography and its NMR and mass spectra confirmed the structure i.e. benzyl alcohol impurity [shown in Figure 3]. It was formed due to incomplete formation of imine and unreacted starting material i.e. aldehyde getting reduced under hydrogenation conditions directly to benzyl alcohol. An appropriate CAPA was suggested for this deviation.

As part of corrective action, entire batch was converted into $\mathrm{HCl}$ salt, desired secondary amine went into aqueous layer and benzyl alcohol remained in organic layer. After layer separation, organic layer was discarded. Product was isolated from aqueous layer after adjustment of $\mathrm{pH}$.

As part of preventive action, additional stirring time of 3 hours was introduced in the process to ensure complete imine formation in pressure reactor.

8. N-oxide impurities: Need for inert atmosphere / using

Ilor grade nitrogen of $99.999 \%$ purity:

In yet another API process transfer, we faced problem due to oxidation of benzimidazole ring of the molecule. Final API was required to be an injectible grade and had slight pale yellow appearance. During downstream processing, molecule was coming in contact with oxygen from atmosphere, hence we used nitrogen blanketing. However, industrial grade nitrogen is not absolutely pure and has some oxygen content in it, due to which we were facing N-oxide impurity issue in final API and appearance of the product was also not as per specifications. Hence, initially, we utilized Ilor grade i.e $99.999 \%$ pure nitrogen gas for blanketing purpose in research lab and later on same grade of nitrogen was employed in scale up and validation campaigns. This completely eliminated issue of N-oxide impurity formation and appearance of API was also improved drastically. Final quality of API as per injectable grade was achieved.

9. Incomplete hydrogenation: reverse hydrogenation,

In a very unusual observation, isoquinolinone was getting dehydrogenated forming an impurity X. This API is antiemetic i.e. used in the treatment of chemotherapy 
induced nausea and vomiting. Since, the hydrogenation was slow and time consuming, we were using almost 50\% $\mathrm{wt} / \mathrm{wt}$ of catalyst loading in proportion with starting material $\mathrm{A}$, in order to hydrogenate it to intermediate $\mathrm{B}$ in a rapid manner. Hydrogenation was fast and complying the "in process" test for starting material content. There was no observation of formation of any impurity during the hydrogenation, in research lab as well as during scale up batch. However, an impurity $\mathrm{X}$ was observed in intermediate $\mathrm{B}$ analysis to the extent of $5 \%$. It was very difficult to remove by normal purification and recrystallisation methods and resulted in huge loss of yield in small experiments conducted during investigations.

After detailed investigation, it was found to be impurity $\mathrm{X}$ as per the mass and ${ }^{1} \mathrm{H}$ NMR data. This was due to reversal of hydrogenation process during filtration and holding of reaction mass for longer time due to slow filtration at nutsch filter in manufacturing plant. Intermediate $\mathrm{B}$, remained in contact with large amount of $\mathrm{Pd} / \mathrm{C}$ catalyst, which dehydrogenated the cyclohexane ring $Z$, resulting in formation of impurity $X$.

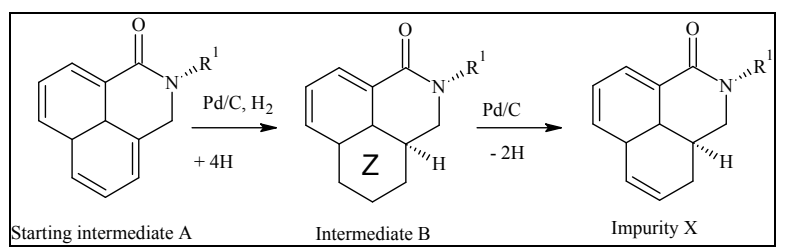

Figure 4. Impurity X formation due to dehydrogenation.

As the material being hydrogenated was very expensive and needed to be supplied to customer in a very tight schedule. Experiments with small quantities were conducted using $10 \% \mathrm{wt} / \mathrm{wt}$ catalyst loading and no dehydrogenation impurity was observed. Based on these findings, corrective action for this batch was proposed and remaining material with impurity $\mathrm{X}$ was hydrogenated using $10 \% \mathrm{wt} / \mathrm{wt}$ catalyst loading and filtered. There was no impurity formation this time and intermediate $\mathrm{B}$ complied the specifications. Finally, in validation campaign, $10 \% \mathrm{Pd} / \mathrm{C} \mathrm{wt} / \mathrm{wt}$ loading was used and completed successfully without any quality issues.

10. Melting point was observed higher than reported in patents, why? As purity goes up the melting point can be improved and can be higher than impure API.

During final API analysis of Ibandronate sodium melting point was coming higher than the reported values in literature [8], Reported values are $175-177^{\circ} \mathrm{C}$ and we obtained in the range of $185-188^{\circ} \mathrm{C}$. Due to such difference in melting points, it was a matter of investigation. Other analyses were confirming purity of product to be $99.99 \%$ by HPLC and titrimetric assays. We concluded that since the product we manufactured was of highest purity, it was showing higher melting point. It is assumed that the product used in literature may not be as pure as we had, and may be impure; hence it had shown less melting point of $175-177^{\circ} \mathrm{C}$. It was also supported by higher melting point values reported in other patents related to Ibandronate sodium.

11. Trans-esterification: addition of IPA at higher temperature, lower the temperature of addition.

In one the API used as antiretroviral, the molecule is having isopropyl di-ester functional group. In final step of salt formation, fumaric acid is dissolved in Isopropyl alcohol at $80^{\circ} \mathrm{C}$ and filtered hot to remove any particulate matter from fumaric acid. This hot solution of fumaric acid in IPA is added to cold solution of free base. An impurity of isopropyl ester of about $0.7 \%$ was observed in final API [USP limit not more than $0.3 \%$ ]. This impurity was formed due to trans-esterification reaction of hot IPA solvent with di-ester function of API. Since the impurity and API are having very similar structure, it could not be removed by normal purification techniques. There was no corrective and preventive action suggestion from $R \& D$. The process could not be validated for regulated markets and the project was abandoned due to this persistent issue.

12. Removal of $\mathrm{NaCl}$ by large quantities water washings. Conductivity of water wash should be below 2000 micro Siemens, then stop washing and go for drying. If conductivity is more than 2000 micro Siemens, API fails in sulphated ash limit [NMT $0.1 \% \mathrm{wt} / \mathrm{wt}$ ] due to $\mathrm{NaCl}$ being trapped in gel of API.

In an API used as phosphate binder, sodium hydroxide was used in the process and later on conc. $\mathrm{HCl}$ was used to adjust $\mathrm{pH}$ as final API was hydrochloride salt. API was a polymer having tendency to swell almost 20 times wt/wt. Due to this property, sodium chloride was getting entrained into the wet gel like API and difficult to wash away during water washings. One of the batch had higher sulphated ash content of about $0.5 \% \mathrm{wt} / \mathrm{wt} \quad$ (limit not more than $0.1 \%$ wt/wt). It was necessary to define exactly how much water wash should be given to wet gel like API, in order to remove sodium chloride from the wet cake/gel. We thought of an innovative way to do away with this issue. We did small experiments in lab for washing wet cake gel and checked the conductance of water wash. It was coming about $5000 \mu \mathrm{S}$. As we continued washings with water, conductance of the water washings fallen below $1000 \mu \mathrm{S}$. We dried samples of wet gel at different stages of washings and generated data for corresponding sulphated ash results[Table 1].

Table 1. showing fall in conductance of water wash and corresponding sulphated ash content.

\begin{tabular}{lll}
\hline $\begin{array}{l}\text { Water wash } \\
\text { volumes }\end{array}$ & $\begin{array}{l}\text { Conductance in } \\
\boldsymbol{\mu S}\end{array}$ & $\begin{array}{l}\text { Sulphated ash content wt/wt } \\
\text { [limit not more than } \mathbf{0 . 1} \%]\end{array}$ \\
\hline 5 volumes & $5000 \mu \mathrm{S}$ & $0.7 \%$ \\
10 volumes & $3000 \mu \mathrm{S}$ & $0.3 \%$ \\
12 volumes & $2000 \mu \mathrm{S}$ & $0.15 \%$ \\
14 volumes & $1000 \mu \mathrm{S}$ & $0.07 \%$ \\
16 volumes & $700 \mu \mathrm{S}$ & $0.03 \%$ \\
\hline
\end{tabular}

Thus, it was necessary to wash API gel with at least 14 to 16 volumes of water with soaking time of about 1 hour 
to allow sodium chloride to diffuse through wet gel and get washed away with water washings. In this issue, we used a simple technique of conductivity and could be classified as an example of PAT i.e. in process analytical technology.

\section{Improvements Done Due to Trouble Shooting and Deviation, As CAPA}

1. Chemistry: Bromination: Benzylic bromination reaction time reduced to $10 \mathrm{hrs}$ from $36 \mathrm{hrs}$.

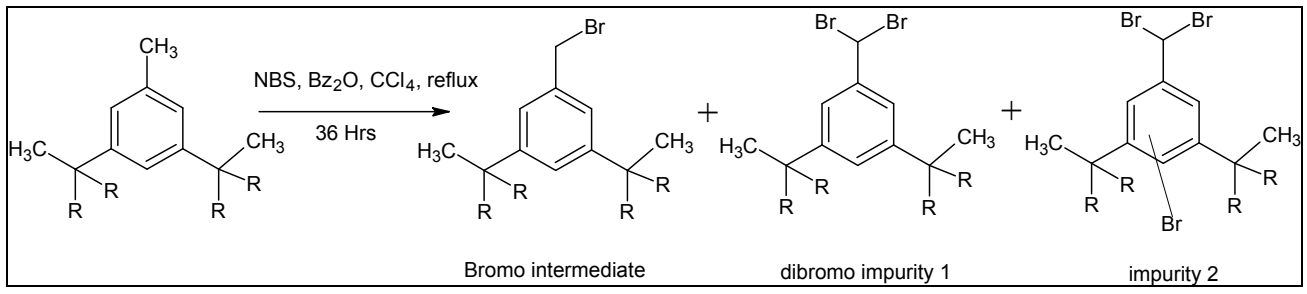

Figure 5. Benzylic bromination \& formation of impurities.

In one of the benzylic bromination process, using NBromosuccinamide, due to extended reaction time of 36 hours, dibromo impurity 1 and impurity 2 were observed. The yield of subsequent coupling product with triazole was very less due to dibromo impurity. Further purification step also reduced the overall yield of the process to just about $15 \%$ [ Figure 5]. This resulted in a deviation due to less yield during scale up trial batches. Less yield implies higher costs of manufacturing and if costs don't come down, generic drug companies are in trouble.

As part of investigation, we reduced the mole equivalents of NBS and reaction time to 10 hours instead of 36 hours, formation of dibromo impurity 1 and impurity 2 was reduced considerably. The yield of further coupling with triazole was also improved upto overall $40 \%$. The number of purifications required were also less compared to earlier process.

2. Chemistry : Chlorination reagent changed from thionyl chloride to phosphorous oxy chloride.

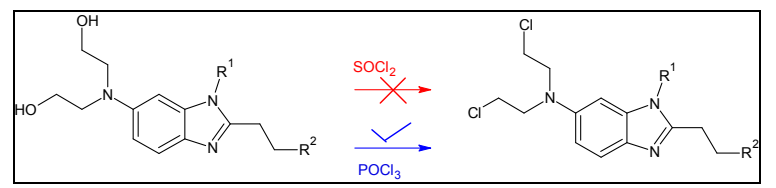

Figure 6. Establishing an alternate reagent for chlorination.

In a process for conversion of aliphatic hydroxyl group to chloro groups in a class of compounds called as Nitrogen mustard drugs for treatment of cancer, we were using thionyl chloride as reagent for chlorination. However, during quenching with water, reaction was very exothermic and vigorous due to evolution of hydrochloric acid gas and sulphur dioxide gas. Due to exothermicity, degradation impurities were observed and 7 to 9 purifications were required resulting into loss of overall yield and process became economically unviable[ Figure 6].

After, unsuccessful scale up trial, we decided to use phosphorous oxychloride as chlorination agent. The quenching with water was vigorous, but under controlled conditions of temperature and stirring, it went on smooth. We observed that the formation of degradation impurities was significantly reduced and quality of crude API was improved to a great extent. With 2-3 purifications, we could achieve purity required for regulatory market i.e. as per ICH guidelines.

3. Equipments: Greenish tinge to API due to contamination/contact with iron metal parts of equipment, replace with PP nutsch filter.

In yet another deviation on appearance of final API, we observed that the API was having greenish tinge in its appearance instead of pure white. It was found that the final solution of API after charcolisation in water came in contact with iron metal parts of a nutsch filter. Hence slight colour was imparted to solution, which ultimately made API having greenish tinge. The nutsch filter with iron bolts was changed to completely PP nutsch filter. This avoided any contact with iron parts and colour of API obtained was pure white.

4. Quality: brown particles due to higher skin temperature, reprocessing procedure is necessary to comply quality appearance specifications.

In an injection grade API used as direct thrombin inhibitor and very costly medicine, brown particles were observed in API powder. It was due to overheating of material due to skin temperature during recovery of solvents on rotavapor. Since recovery of the solvent was part of process and could not be avoided, we introduced a reprocessing procedure in another solvent and charcolisation to get rid of brown particles by way of filtration. Final API obtained was white and had better clarity of solution as per specifications of injectible grade API.

5. Quality/Process: MDC solvent specification NMT $600 \mathrm{ppm}$ as per ICH limits for residual solvents were not complying in final API, hence IPA stripping was introduced to remove MDC solvent, During IPA stripping, MDC solvent was removed effectively, crude API was isolated in MTBE and then purified in IPA.

In a process, methylene chloride solvent was used and it was distilled off during isolation procedure. It was observed in residual solvent analysis that API was failing in MDC content of about 1400ppm. Further drying of API was not effective, as MDC content remained on higher side. 
Due to tighter limit of MDC content in Drug substance [ ICH guideline, Q3 limit of MDC not more than 600ppm]. We had to decide to introduce an IPA stripping after recovery of MDC solvent. Degassing was done for almost two hours under vaccum and $50 \mathrm{~mm} / \mathrm{Hg}$ vacuum and then IPA solvent stripping was introduced to drive off residual MDC solvent in semisolid material.

6. Purge of impurity : An Unknown impurity had a limit of $0.3 \%$ and in process test result was $0.29 \%$, it was failing on border. Hence a deviation done and batch processed further. Final API was passing, as this impurity was purged during further isolation steps in the process.

7. Chromatography: delayed fraction collection

In an oncology API having low volume, high cost scenario, we were purifying crude API using preparative HPLC technique. The fraction collection was done using an automated state of the art fraction collector. However, we were encountered with an unknown impurity in initial fractions. Fraction 1 being the most impure was discarded. Most of the material was isolated from initial fractions i.e. fraction 2,3,4 \&5, which were not passing specification for an unknown impurity. This unknown impurity was not separated/ resolved properly in prep-HPLC column and was getting merged or co-elutes with the main peak/ compound.

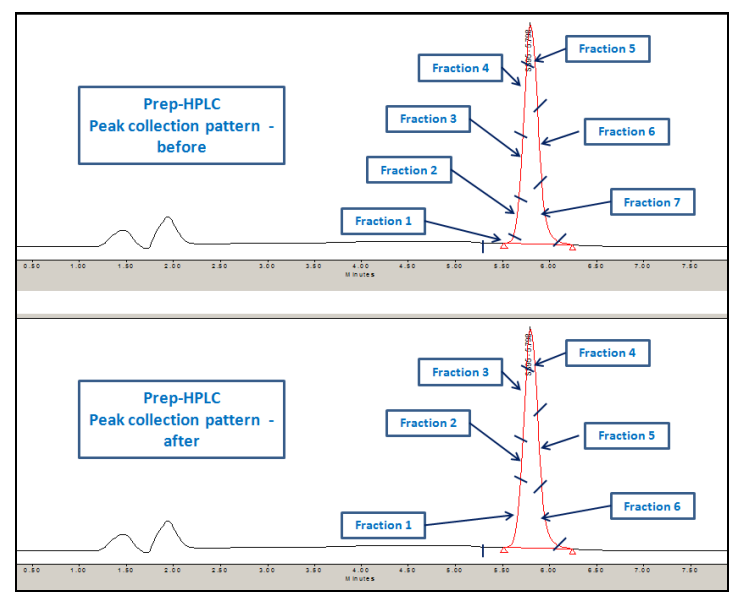

Figure 7. Preparative HPLC \& delayed fraction collection to achieve purity.

We decided to do a simple trick while collecting fractions of prep-HPLC column. As shown in Figure 7, we delayed the collection of fractions by about 30 seconds, and collected fraction $2,3,4 \& 5$ as shown in figure. Since fraction 1 was now collected for additional 30 seconds, most of the impurity was eluted in this fraction. Fraction 2, $3,4 \& 5$ were analysed individually by HPLC and found to be complying with respect to unknown impurity. Finally these fractions were mixed and API was isolated with about $10 \%$ lesser yield than previous time frame. However, quality of the API was improved significantly to the extent of $0.04 \%$ of unknown impurity.

8. Oxime reduction using pyridine borane, reagent assay might go down due to prolonged storage at ambient temperature. Hence additional reagent alongwith IPA. $\mathrm{HCl}$ needed to be added in order to complete reaction.

Raw material may get degraded over prolonged storage period and higher temperature fluctuations due to climatic conditions in Indian subcontinent. In one of the postvalidation campaign, we found during raw material checking that pyridine.borane was decomposed. QC chemist was not able to withdraw a sample for reanalysis, as the chemical inside a metal drum had decomposed and polymerized. It was noticed, just few days before the actual start of post-validation campaign. Since this raw material was failing in quality, we had to procure fresh from European supplier. The lead time for such supply is 90 days, as the material is not allowed by Air route and has to come all the way by Sea route. Our post-validation campaign had to be postponed till availability of raw material.

9. Quality: XRPD not matching due to low resolution instrument, needs high resolution instrument to decide about polymorphism contamination issues.

In a project having polymorphic API, a low resolution table top model of XRD was used. XRD plot was showing very broad peaks and it was not possible to detect other polymorph due to broad peaks. It was a very specific patent situation that there should not be any contamination from the patent infringement point of view. Hence, samples were sent to an outside agency ( CIPRA, Hyderabad) having high resolution XRD instrument. Later on it was confirmed by quantitative methods that samples were polymorphically pure and there was no contamination of the other polymorph and hence issue of patent infringement was not possible. In this case, we were totally dependent on an outside agency for XRD support, which results in delays.

10. Chemistry: over reduction due to prolonged stirring, Critical Process parameter, send sample for information [GAT guideline for additional testing, ]

During investigations, mainly four parameters i.e. man, machine, material and method are checked to find out possible cause for failure. Preventative action is possible using many tools like FMEA( failure mode effect analysis), fish bone or Ishikawa diagram, FTA (Failure tree analysis) etc. These tools allow for analysis to be branched in order to provide multiple root causes. Corrective action can then be taken to prevent further failures of a similar kind. In a nut shell, we can classify the difficulties faced during transfer of technologies as follows:

1. Raw material related issues

2. In process test related issues

3. Analysis related issues- Intermediate, API analysis issues

4. Operations related

5. Human error during operations

Importance of $Q b D$ : Here we would like to stress the importance of QbD i.e quality by design, a concept uphold by FDA for efficient product development methods/ processes. Above troubleshooting examples described 
would give an idea about what may happen during transfer of API technology. Therefore, a development scientist must keep in mind the probable troubles/ issues and incorporate such controls in the process. The more logical controls we incorporate in the process, better results/outcomes we get and we have better control on quality and cost of the process. The process developed keeping QbD principles in mind would have very rare chance of failure. Processes optimised using QbD principles would have improved process understanding, improved product quality and reduced variability in API manufacturing operations.

\section{Conclusion}

In this paper, we have described various aspects of API technology transfer like trouble shooting, quality issues, safety issues, environmental protection related issues and about CAPA and QbD. We would like to emphasis here that various professionals working in various organization might be facing or might already have faced such or similar issues. Our intentions of making this publication are for information purpose to those who are new entrants in this field and may serve as mere guide for possible corrective and preventive actions in future. It also can serve as a reference paper for professionals seeking to take challenging assignments in API technology transfer domain. It also can be a guiding article to those working in $R \& D$, ADL laboratories to start a better thought process before actually transferring knowledge to commercial production site.

\section{Acknowledgements}

We deeply thank Dr. R. Atram, Principal of I.Y. College, Jogeshwari, Mumbai and the management of Motilal Zhunzhunwala College of Arts, Commerce and Science, Vashi, Navi Mumbai for constant encouragement throughout this work.

\section{References}

[1] Chronicle Pharmabiz, 4th July 2013

[2] K.B. Saji, Karuna Jain, Int. J. of Technology Transfer and Commercialisation, 2006 Vol.5, No.3, 251 - 262.

[3] Binay Kumar Pattnaik, Technology Transfer and In-House R\&d in Indian Industry, Volume - 1999

[4] J. C. Copplestone, Dr. C. M. Kirk , S. L. Death, N. G. Betteridge, S. M. Fellows, Heather Wansbrough, http://nzic.org.nz/ChemProcesses/production/1A.pdf.

[5] ICH Topic Q3C (R4), Impurities: Guideline for Residual Solvents

[6] http://www.aioh.org.au/downloads/documents/ControlBandi ngBNaumann.pdf

[7] http://www.coolpack.com/admin/documents/MCATransport Temp20Med.pdf

[8] (a) Muhlbauer, R.C., et al., J. Bone Miner. Res., 6, 1003 (1991). (b) Pecherstorfer, M., et al., J. Clin. Oncol., 14, 268 (1996). 